\title{
DETERMINAÇÃO DO GRAU DE MATURAÇÃO DO ABACATE (Persea americana Miller), ATRAVÉS DA UTILIZAÇÃO DO PRESSÓMETRO*
}

\author{
ANTONIO A. LUCCHESI ** \\ HEITOR W.S. MONTENEGRO***
}

\section{RESUMO}

Um experimento foi realizado com o objetivo de avtriguar o valor do pressômetro (Fruit Pressure Testers) na determinação do grau de maturação do abacate. Foram estudados frutos de três regiões ecologicamente diferentes do Estado de São Paulo (Novo Horizonte, Limeira e Itapetininga).

Em cada região, utilizou-se de três pomares e em cada pomar selecionou-se cinco árvores das cultivares 'Wagner', 'Prince' e 'Collinson', colhendo-se dois frutos de cada árvore, que no conjunto (10 frutos) formaram a amostra a ser analisada, para cada cultivar.

Efetuou-se colheitas em épocas predeterminadas visando a análise das variações da dureza da polpa de fruto durante o seu desenvolvimento, desde a sua formação até a época de maturação.

Utilizando-se do pressômetro, observou-se um aumentc gradativo da dureza da polpa com o desenvolvimento do fruto, provavelmente devido a diminuição constante de umidade da polpa.

Apesar de se constatar índices diferentes para os diversos estágios de desenvolvimento do fruto, essas diferenças não foram suficientemente amplas de modo a permitir um seguro critério de maturação para o abacate. Constatou-se nâo obstante, condições mais favoráveis para a utilização do pressômetro em frutos produzidos em regiões mais frias, onde se obtém índices mais amplos, nos diversos estágios de desenvolvimento do fruto.

Foi constatado em todas as cuitivares testadas, no estágio inicial do desenvolvimento do fruto, diferenças entre os índices obtidos nas três regiões estudadas, observando-se índices mais altos nas regiōes mais quentes.

\section{INTRODUÇÃO}

Segundo MONTENEGRO (1960), em nosso país não existe até agora nenhuma legislação que regule a colheita de abacates, a exemplo do que se verifica na laranja. $\mathrm{Na}$ Califórnia, EUA, foi estabelecido como mínimo exigido para venda, o teor de $8 \%$ de óleo na polpa do abacate. No Brasil, esse índice de maturação não é possivel de se apli-

* Entregue para publicação em 3/12/1975. Parte da Tese apresentada pelo primeiro autor para a obtenção do título de Doutor na ESALQ/USP.

** Departamento de Botânica - ESALQ/USP.

** Departamento de Agricultura e Horticultura - ESALQ/USP. 
car, pois, são cultivadas em larga escala, cultivares da raça antilhana; que possuem abaixo teor em óleo.

MONTENEGRO (1960) ainda cita que na prática pode-se estimar, por diferentes meios, o ponto de maturação do abacate, para colheita, no estágio conhecido como "de vez". Tais meios são: nas cultivares de frutos com casca de cor roxa, isso se dá quando eles começam a apresentar pequenas áreas de cor arroxeadas; nas de frutos do tipo manteiga (antilhano), quando se verifica mudanças na tonalidade da cor verde para amarelada; outro indício è a facilidade com que se quebra o pedúnculo quando forçado a curvar-se. Em algumas cultivares, como no caso da 'Nimlioh', conhece-se o "ponto" de maturação, porque a semente fica solta dentro do fruto.

Com relação a época do ano, não de pode tomá-la como indicação para se proceder a colheita, segundo MONTENEGRO (1960), pois, de conformidade com as variações bastante comuns de clima que ocorrem todos os anos, a maturação do abacate pode adiantar-se, ou atrasar-se.

Pelo visto, não é fácil determinar, de maneira prática, o ponto de colheita do abacate. Em algumas frutíferas, tem sido utilizado o pressômetro (Fruit Pressure Testers) que mede a resistência que a polpa do fruto oferece à penetração (HALLER, 1941), avaliando-se através da utilização desse aparelho, o gradual amadurecimento dos frutos.

$O$ presente trabalho tem a finalidade de testar a utilização desse aparelho para uma possível determinação do grau de maturação do abacate.

\section{REVISÃO DA LITERATURA}

Através da utilização do pressômetro para avaliar o gradual amadurecimento do fruto, muitos trabalhos foram executados, com bons resultados, como: em maçã (HALLER et alii, 1941); banana (DEULLIN, 1952 e DEULLIN \& MONNET, 1956); ameixa (WILEY \& WORTHINGTON, 1955); pêra (D'ERSU \& SWINZOW, 1955); pêssego (ROOD, 1957 e DUPAIGNE, 1961); abricó (D'ERSU \& SWINZOW, 1955 e DUPAIGNE, 1961); morango (HALLER et alii, 1932), entre outros.

Em abacate, segundo STAHL (1933), a utilização do pressômetro em cultivares na Flórida, foi impraticável.

A essa mesma conclusão também chegou IZURIETA (1970), trabalhando com algumas cultivares no Estado de São Paulo.

ULRICH (1952) diz que a dureza dos frutos medida com um dinamômetro, dimiriui durante a maturação. Hallen, citado por ULRICH (1952), diz que a dureza dos frutos está relacionada com as condições locais de clima (temperatura, umidade), com o porta-enxerto, com a riqueza em nitrogênio, entre outros fatores.

Segundo Magness \& Burroughs, citado por ULRICH (1952), o amolecimento da polpa do fruto é mais rápido em temperaturas mais elevadas.

ULRICH (1952) cita que a maturação é caracterizada, na maioria dos frutos por uma queda progressiva da dureza; e a dureza dos frutos está relacionada principalmente com o teor de pectina, espessura das paredes celulares, e turgescência das células. 
O mesmo autor diz que, durante o seu desenvolvimento na planta, os frutos carnosos se enriquecem bastante em água; no entanto, existe exceções, como no caso da pêra, na qual de $83 \%$ de água, quando verde, passou a $79 \%$ quando madura.

HALLER (1941) verificou que à medida que o fruto se desenvolve na árvore, ou amadurece após a colheita, há um gradual amolecimento da polpa. $\mathrm{O}$ amolecimento do fruto após a colheita tem sido relacionado com a hidrólise do material cimentante insolúvel da parede celular, e com a transformação de protopectina para pectina solúvel (HALLER, 1941 e BIALE, 1970).

Segundo SACHER (1962) nessa fase, há marcantes alterações, na permeabilidade da casca e dissociação celular (separação de células na lamela média).

Segundo BIALE \& YOUNG (1962) quando um abacate totalmente formado, é retirado da árvore e colocado em condições favoráveis para a maturação, a intensidade respiratória declina até alcançar o mínimo, em alguns dias ou semanas, segundo seu grau de desenvolvimento e as condições de temperatura e aeração. Em continuação é produzida uma repentina e marcada reativação respiratória, denominada "reativação climatérica". Climatérico, segundo os mesmos autores, pode-se considerar como a fase na vida dos frutos que separa o desenvolvimento e maturação, da senescência; durante este estágio, se produzem marcadas trocas na resistência às doenças, nas características celulares e nas reações metabólicas, todos os quais conduzem à senescência do fruto. $\mathrm{O}$ abacate, não é comest ível antes que o climatérico alcance seu ponto máximo. Relacionando-se os estágios de fruto colhido (totalmente desenvolvido, mas a polpa dura) e de fruto maduro (depois de armazenado, polpa mole), há diminuição nos açúcares totais; aumento no teor de graxas e de proteínas (BIALE \& YOUNG, 1962).

Segundo BURG \& BURG (1962) e LEOPOLI) (1964), no abacate, só começará o climatérico depois de ser colhido da árvore. Por ui razão os frutos não amolecem enquanto permanecem na árvore.

\section{MATERIAIS E MÉTODOS}

No período de dezembro de 1972 a novembro de 1973, colheu-se frutos de três cultivares de abacate - 'Wagner', 'Prince' e 'Collinson', em pomares de três regiơes do Estado de São Paulo: Novo Horizonte, Limeira e Itapetininga, com o objetivo de determinar o estágio adequado de colheita, com vistas a um maior rendimento industrial.

Em cada região estudada foram selecionados três pomares que receberam as denominações de pomar A, B e C nos quais existem as cultivares estudadas: 'Wagner', 'Prince' e 'Collinson'.

Dentro de cada pomar foram selecionadas cinco árvores de cada cultivar com bom desenvolvimento vegetativo e em boas condições fitossanitárias. De cada árvore foram colhidos dois frutos dentre os mais desenvolvidos, que no conjunto (dez frutos) formaram a amostra a ser analisada de cada cultivar.

A colheita dos frutos foi realizada nas datas constantes no Quadro 1, visando a análise do desenvolvimento do fruto, desde o início de sua formação até o estágio de maturação completa. 
QUADRO 1 - Datas de colheita do fruto das cultivares estudadas.

\begin{tabular}{lccc}
\hline Regiōes & 'Wagner' & 'Rrince' & 'Collinson' \\
\hline \hline \multirow{3}{*}{ Novo Horizonte } & $13 / 12 / 72$ & $13 / 12 / 72$ & $13 / 12 / 72$ \\
& $31 / 01 / 73$ & $31 / 01 / 73$ & $31 / 01 / 73$ \\
& $28 / 03 / 73$ & $28 / 03 / 73$ & $28 / 03 / 73$ \\
& $23 / 05 / 73$ & $23 / 05 / 73$ & $23 / 05 / 73$ \\
& $23 / 08 / 73$ & $23 / 08 / 73$ & - \\
& & & $05 / 12 / 72$ \\
Limeira & $05 / 12 / 72$ & $05 / 12 / 72$ & $30 / 01 / 73$ \\
& $30 / 01 / 73$ & $30 / 01 / 73$ & $18 / 04 / 73$ \\
& $18 / 04 / 73$ & $18 / 04 / 73$ & $14 / 06 / 73$ \\
& $14 / 06 / 73$ & $14 / 06 / 73$ & - \\
Itapetininga & $04 / 09 / 73$ & $04 / 09 / 73$ & $12 / 12 / 72$ \\
& $12 / 12 / 72$ & & $06 / 03 / 73$ \\
& $06 / 02 / 73$ & $12 / 12 / 72$ & $16 / 05 / 73$ \\
& $16 / 05 / 73$ & $06 / 02 / 73$ & $16 / 07 / 73$ \\
\hline
\end{tabular}

Após a colheita, os frutos etiquetados, e acondicionados cuidadosamente em sacos de polietileno, foram levados ao laboratório do Departamento de Tecnologia dos Alimentos da Escola Superior de Agricultura "Luiz de Queiroz", em Piracicaba, SP, onde determinou-se a umidade natural da polpa, utilizando-se estufa Thelco, modelo 18, com circulaçăo de ar quente, à temperatura de 105 a $110^{\circ} \mathrm{C}$, até peso constante; e balança Mettler P $1200 \mathrm{~N}$ com precisão de 0,01 grama.

O pressômetro utilizado na de terminação do grau de dureza da polpa do abacate é do tipo $10 \mathrm{~B}$, da D. Ballaufmfg. Co. Inc. Washington, U.S.A., e a ponta perfuradora, testada para os frutos de abacate e utilizada no trabalho, tem $4 \mathrm{~mm}$ de diâmetro por $25 \mathrm{~mm}$ de comprimento. Para isso, retirou-se uma secção de aproximadamente 4 centímetros quadrados de casca, na região mediana do fruto, no centro do qual se testou a dureza. Após a perfuração, foi lido na escala, o índice de resistência que a polpa oferecia à penetração (Fig. 1).

O pressômetro, segundo Magness \& Taylor, citado por HALLER (1941) consiste em um tubo de metal cilíndrico dentro do qual trabalha um êmbolo fixo ao cilindro por $u$ ma mola espiral de aço sob tensão. A calibração, de 0 a 10 , de meia em meia unidade, é em libras de pressão, necessárias para forçar o êmbolo para trás. Na parte anterior do aparelho, há uma ponta perfuradora que penetra na polpa do fruto até o limite de uma roela (Fig. 1). 


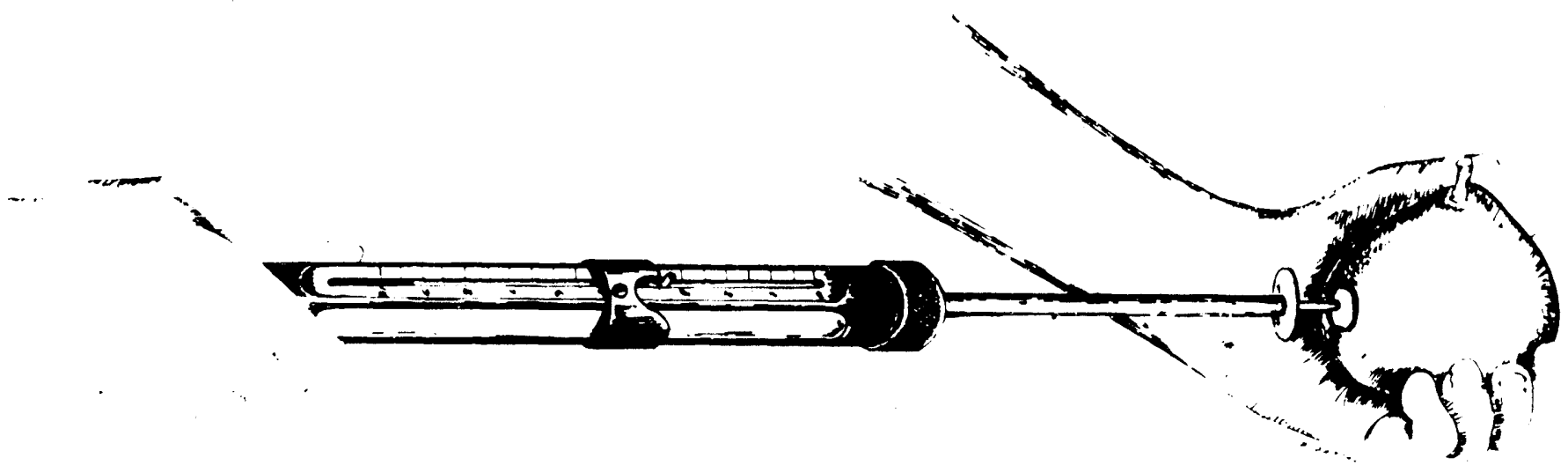

FIG. 1 - O aparelho (pressômetro) utilizado.

Segundo FERREIRA et alii (1957), os parâmetros climáticos das regiões estudadas são os seguintes:

Novo Horizonte: altitude de 453 m; 21028'02" de latitude sul e 49013'17" de longitude W.Gr.

Limeira: altitude de 542 m; 22033'49" de latitude sul e 47024'15" de longitude W.Gr.

Itapetininga: altitude de $670 \mathrm{~m}$; 23035'09" de latitude sul e 48002'51" de longitude W.Gr.

Do ponto de vista climático, segundo a Classificação do Clima, pelo Sistema Inter. nacional de Koeppen (SETZER, 1966), as regiões estudadas, possuem as seguintes características:

Novo Horizonte: Aw - clima tropical com estiagem no inverno, com menos de $30 \mathrm{~mm}$ de chuva no mês mais seco, sendo a temperatura média do mês máis quente acima de $22^{\circ} \mathrm{C}$ e do mês mais frio acima de $180 \mathrm{C}$.

Limeira: $\mathrm{Cw}$ - clima temperado com estiagem no inverno, com menos de $30 \mathrm{~mm}$ de chuva no mês mais seco, sendo a temperatura média do mês mais quente acima de $22{ }^{\circ} \mathrm{C}$ e do mês mais frio abaixo de $18{ }^{\circ} \mathrm{C}$.

Itapetininga: $\mathrm{Cf}$ - clima temperado sem estação seca, com mais de $30 \mathrm{~mm}$ de chuva no mês mais seco, sendo a temperatura média do mês mais quente acima de $220 \mathrm{C}$ e do mês mais frio, abaixo de $180 \mathrm{C}$.

Segundo a COMISSÃO DE SOLOS (1960), os solos das regiões estudadas são:

Novo Horizonte: Podzólico Vermelho-Amarelo "Intergrade" para Latossol VermelhoAmarelo.

Limeira: Solos Podzolizados de Lins e Marília, variação Lins.

Itapetininga: Latossol Vermelho-Escuro Orto. 


\section{RESULTADOS E DISCUSSÃo}

A análise estatística dos dados obtidos (média de 10 frutos) foi efetuada através de análise de variância em blocos casualizados. Os dados são dos índices obtidos no pressômetro (em libras de pressão), durante o desenvolvimento do fruto, em cada região.

QUADRO 2 - Leitura do pressômetro, na região de Novo Horizonte.

\begin{tabular}{|c|c|c|c|c|c|c|c|c|c|c|}
\hline \multirow{2}{*}{\multicolumn{2}{|c|}{$\underbrace{\text { Cultivar }}_{\text {Pomares }}$}} & \multicolumn{3}{|c|}{ 'Collinson' } & \multicolumn{3}{|c|}{ 'Prince' } & \multicolumn{3}{|c|}{ 'Wagner' } \\
\hline & & & & & & & & & & \\
\hline \multicolumn{2}{|c|}{ Data } & $\mathbf{A}$ & B & $\mathrm{C}$ & A & B & $\mathrm{C}$ & A & B & $\mathrm{C}$ \\
\hline $1 \mathrm{a}$ & $13 / 12 / 72$ & 9,0 & 8,5 & 8,5 & 7,1 & 7,3 & 6,7 & 8,4 & 8,5 & 8,3 \\
\hline $2 \mathrm{a}$ & $31 / 01 / 73$ & 9,4 & 9,5 & 9,4 & 7,8 & 7,6 & 7,7 & 9,1 & 9,0 & 9,3 \\
\hline $3 a$ & $28 / 03 / 73$ & 9,5 & 9,6 & 9,5 & 8,0 & 7,8 & 7,9 & 9,4 & 9,4 & 9,5 \\
\hline $4 a$ & $23 / 05 / 73$ & 9,7 & 9,6 & 9,6 & 8,2 & 8,4 & 8,3 & 9,7 & 9,5 & 9,6 \\
\hline $5 a(*)$ & $23 / 08 / 73$ & - & - & - & 9,6 & 9,6 & 9,5 & 9,6 & 9,5 & 9,8 \\
\hline
\end{tabular}

(*) Nas análises, não foi computada a 5 ạ data, pois já não havia mais frutos da cultivar 'Collinson', que é mais precoce que as outras duas cultivares.

A primeira data difere de todas; as outrras não diferem entre si.

QUADRO 3 - Leitura do pressômetro na regiâo de Limeira.

\begin{tabular}{|c|c|c|c|c|c|c|c|c|c|c|}
\hline \multirow{2}{*}{\multicolumn{2}{|c|}{$\underbrace{\text { Cultivar }}_{\text {Pomares }}$}} & \multicolumn{3}{|c|}{ 'Collinson' } & \multicolumn{3}{|c|}{ 'Prince' } & \multicolumn{3}{|c|}{ 'Wagner' } \\
\hline & & & & & & & & & & \\
\hline \multicolumn{2}{|c|}{ Data } & A & B & $\mathrm{C}$ & A & B & $\mathrm{C}$ & A & B & $\mathrm{C}$ \\
\hline $1 \mathrm{a}$ & $05 / 12 / 72$ & 7,8 & 7,7 & 7,7 & 6,5 & 6,7 & 6,4 & 8,2 & 8,2 & 8,0 \\
\hline $2 \mathrm{a}$ & $30 / 01 / 73$ & 8,8 & 8,6 & 8,5 & 7,5 & 7,4 & 7,5 & 8,3 & 9,0 & 9,0 \\
\hline $3 \mathrm{a}$ & $18 / 04 / 73$ & 9,3 & 9,2 & 9,4 & 7,7 & 7,6 & 7,8 & 9,1 & 9,2 & 9,3 \\
\hline $4 \stackrel{a}{a}$ & $14 / 06 / 73$ & 9,7 & 9,5 & 9,7 & 8,1 & 8,1 & 8,0 & 9,5 & 9,5 & 9,6 \\
\hline $5 \mathrm{a}$ & $04 / 09 / 73$ & - & - & - & 9,5 & 9,4 & 9,6 & 9,6 & 9,5 & 9,5 \\
\hline
\end{tabular}


A primeira e a segunda data diferem entre si e das outras, mas a partir da terceira não há diferença significativa.

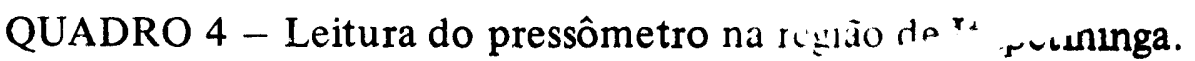

\begin{tabular}{|c|c|c|c|c|c|c|c|c|c|c|}
\hline \multicolumn{2}{|c|}{ Cultivar } & \multicolumn{3}{|c|}{ Collinson' } & \multicolumn{3}{|c|}{ 'Prince' } & \multicolumn{3}{|c|}{ 'Wagner' } \\
\hline \multicolumn{11}{|c|}{ Pomares } \\
\hline Dat & & A & B & $\mathrm{C}$ & $\mathrm{A}$ & B & $\mathrm{C}$ & A & B & $\mathrm{C}$ \\
\hline $1 \mathrm{a}$ & $12 / 12 / 72$ & 6,8 & 7,1 & 7,0 & 6,3 & 6,1 & 6,0 & 8,0 & 7,7 & 7,5 \\
\hline $2 \mathrm{a}$ & $06 / 02 / 73$ & 8,3 & 8,4 & 8,1 & 7,3 & 7,5 & 7,5 & 8,2 & 8,6 & 8,4 \\
\hline $3 a$ & $16 / 05 / 73$ & 8,5 & 8,7 & 8,6 & 7,7 & 7,7 & 7,6 & 8,7 & 8,8 & 8,7 \\
\hline $4 a$ & $16 / 07 / 73$ & 8,7 & 9,1 & 9,2 & 8,2 & 8,2 & 8,4 & 9,3 & 9,5 & 9,4 \\
\hline 5 a & $07 / 11 / 73$ & - & - & - & 9,4 & 9,4 & 9,5 & 9,4 & 9,6 & 9,5 \\
\hline
\end{tabular}

As diferenças foram mais evidentes entre a primeira data e as outras. Para a cultivar 'Wagner' houve diferença significativa entre a terceira e a quarta datas.

$\mathrm{O}$ valor de $\mathrm{F}$ para Blocos (Pomares) não foi significativo nas três regiões estudadas.

Os Gráficos 1 e 2 apresentam a evolução verificada durante o desenvolvimento do abacate, desde a sua formação até a maturação final. Os valores utilizados nos gráficos são média dos três pomares estudados em cada região.

Segundo ULRICH (1952) e HALLER (1941), de um modo geral, em fruticultura, à medida que o fruto se desenvolve na árvore, há um gradual amolecimento da polpa, em decorrência do aumento da umidade. No presente trabalho, como se observa no Gráfico 1 , o teor de água na polpa do abacate, diminui com o desenvolvimento do fruto, e provavelmente, em conseqüência a isso, há um endurecimento gradativo, e portanto, uma maior resistência da polpa ao pressômetro, constatando-se índices cada vez maiores (Quadros 2 a 4; e Gráfico 2), até o estágio maduro para consumo, momento em que a polpa já não possui mais nenhuma resistência ao pressômetro. Essa perda de turgência é justificada por SACHER (1962) da seguinte maneira: há nessa fase, marcantes alterações na permeabilidade da casca e dissociação celular (separação de células na lamela média). O climatérico, segundo BURG \& BURG (1962) e LEOPOLD (1964) só se dará depois do fruto ser colhido da árvore, por essa razão o fruto não amolece enquanto permanecer na árvore.

Na região mais quente, Novo Horizonte, por ocasião da primeira colheita, se constatou que o fruto já estava em estágio de desenvolvimento mais avançado, e portanto, apresentando índices maiores no pressômetro (Quadro 2), enquanto que na região mais fria, Itapetininga, partiu-se de estágio menos avançado de desenvolvimento, obtendo-se conseqüentemente, índices mais baixos no pressômetro (Quadro 4).

Através dos dados obtidos, observa-se que quanto mais fria a região, mais lenta é a evolução da perda de água da polpa, ao ponto de terem sido constantes maior número de diferenças significativas entre épocas de colheita na região mais fria, e quase nenhuma significância na região mais quente. 
414

Anais da E.S.A. "Luiz de Queiroz"
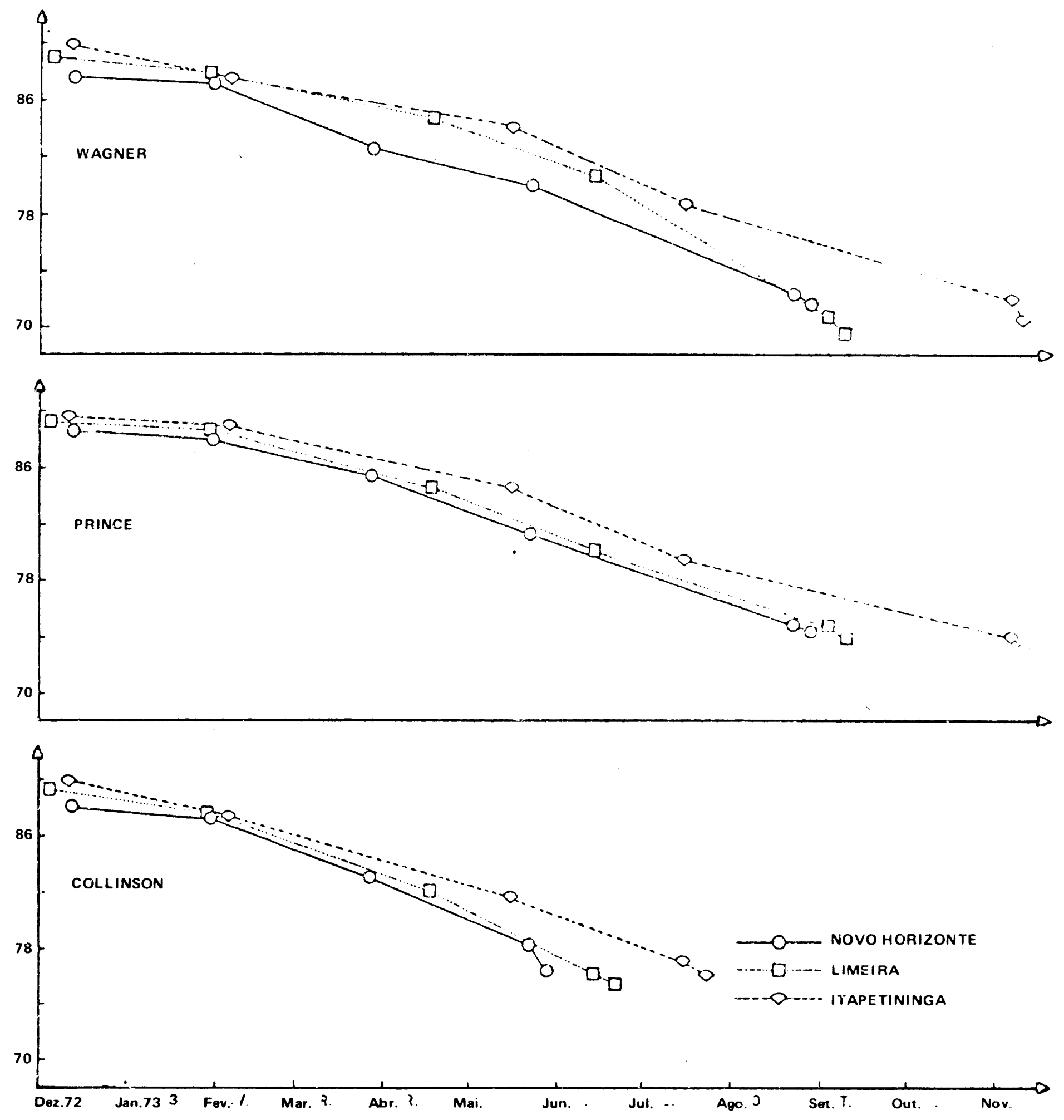

GRÁFICO 1 - Porcentagem de água na polpa. 

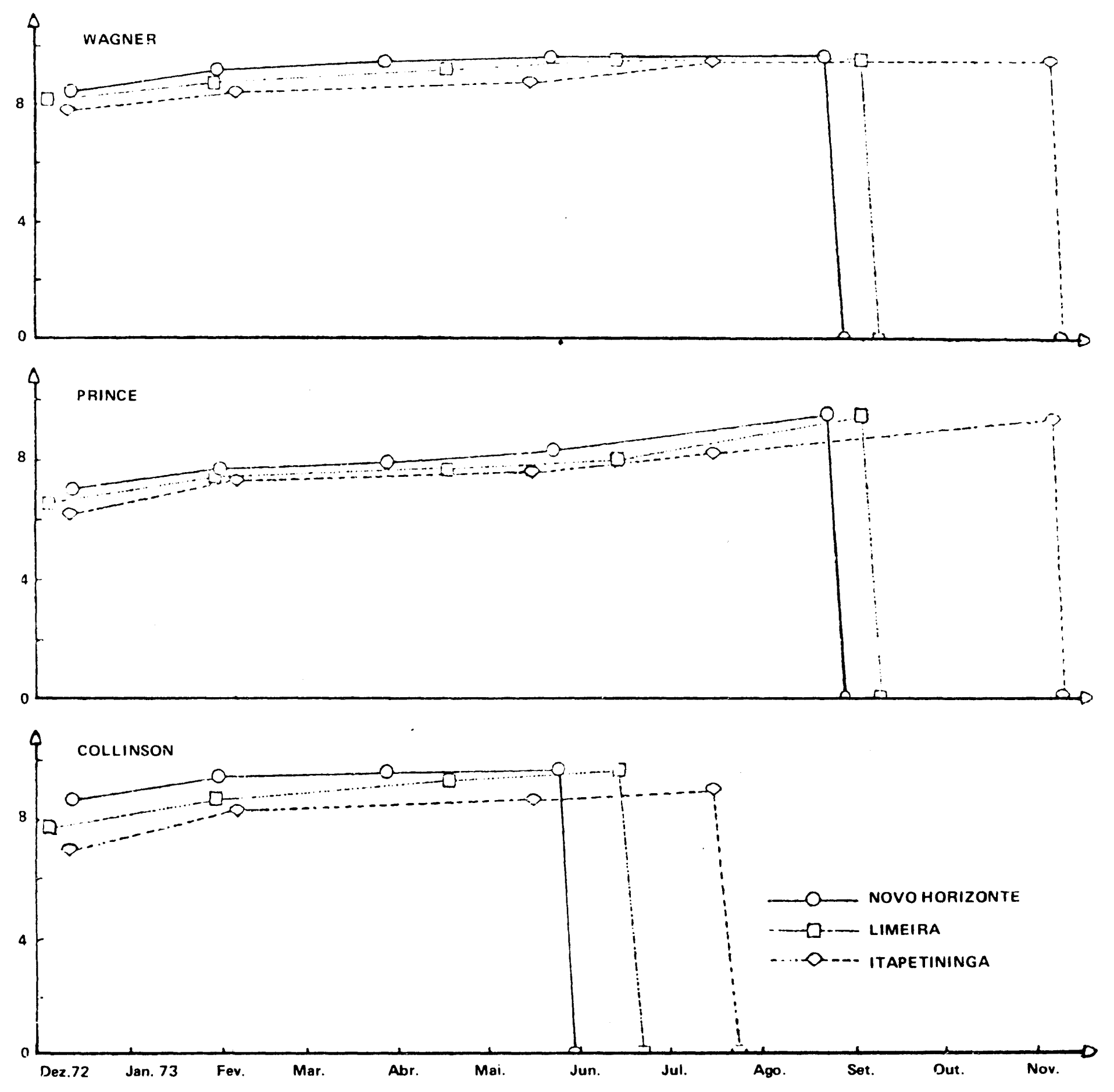

GRÄFICO 2 - Resistência da polpa à pene tração (leitura do pressômetro, em libras). 
Do exposto, observa-se que apesar de se constatar índices diferentes para os diversos estágios de desenvolvimento do fruto, essas diferenças não são suficientemente åmplas de modo a permitir um seguro critério de maturação para o abacate. Não obstante, constata-se condições mais favoráveis para a utilização do pressômetro em frutos produzidos nas regiões mais frias, onde se obtém índices mais amplos, nos diversos estágios de desenvolvimento do fruto.

\section{CONCLUSÕES}

1 - A dureza da polpa do abacate e por conseqüência a sua resistência à penetração do pressômetro aumenta com o desenvolvimento do fruto, provavelmente devido à diminuição constante de umidade da polpa.

2 - Apesar de se constatar índices diferentes para os diversos estágios de desenvolvimento do fruto, essas diferenças não foram suficientemente amplas de modo a permitir um seguro critério de maturação para o abacate.

3 - Constatou-se, não obstante, condições mais favoráveis para a utilização do pressômetro em frutos produzidos em regiões mais frias, onde se obtém índices mais amplos, nos diversos estágios de desenvolvimento do fruto.

4 - Constatou-se em todas as variedades testadas, no estágio inicial do desenvolvimento do fruto, diferenças entre os índices obtidos nas três regiōes, observando-se índices mais altos nas regiões mais quentes.

\section{SUMMARY}

\section{DETERMINATION OF MATURATION DEGREE OF AVOCADO (Persea ameri- cana MILLER) THROUGHT OUT UTILIZATION OF FRUIT PRESSURE TESTERS}

An experiment was realized to verify the value of fruit pressure tester in the determination of avocado maturation degree. It was utilized fruits of three ecologicaliy different regions of São Paulo State, Brazil.

From each region, three orchards were studied. It was selected five trees of 'Wagner', 'Prince' and 'Collinson' cultivars in each orchard. It was picked up two fruits of each three making a ten fruits set for each cultivar to be analysed.

Harvestings were done in pre-determined time to analyse variations of fruit pulp toughness during its development from setting to maturation (ripening).

It was observed a gradative increase of pulp tougness with fruit development utilizing fruit pressure testers, probably due to constant decreasing of pulp humidity.

Althrough of different indexes for various stages of fruit development, these differences were not wide enough to gave a safe criterion of avocado maturation. But, 
there were better conditions of fruit pressure testers utilization for fruits produced in cooller regions where it can get wider indexes in various stages of fruit development.

In early stage of fruit development it was evidence $u$ in all tested cultivars differences between obtained indexes in all three studied regions, being higher indexes in warmer regions.

\section{LITERATURA CITADA}

BIALE, J.B. \& YOUNG, R.E., 1962. Bioquímica da la maturación de los frutos. Endeavour. Londres, $21(83 / 84): 164-174$.

-, 1970. The ripening of fruit. IN: Plant Agriculture. Scientific American, W.H. Freeman and Company San Francisco, 70-74.

BURG, S.P. \& BURG, E.A., 1962. Post-harvest ripening of avocados. Nature. MacMillan and Co. Ltd., New York, 194(4826):398-399.

COMISSĀO DE SOLOS, 1960. Levantamęnto de reconhecimento de solos do Estado de São Paulo. Serv. Nac. Pesq. Agron., Bol. nọ 12, Min. Agric. CNEPA. Rio de Janeiro, 1-634.

D'ERSU, P. \& SWINZONW, H., 1955. Evolution des abricots Canino. Fruits, I.F.A.C., France, 10(10):469-481.

DEULLIN, R., 1952. La possibilité de mieux connaitre le degré d'evolution de la banane. Fruits, I.F.A.C., France, 7(11) :529-533.

\& MONNET, J., 1956. La dureté de la banane pendant le developpment et en phase preclimatérique. Fruits, I.F.A.C., France, 11(8):341-354.

DUPAIGNE, P., 1961. Sur la détermination de la fermeté des fruits au moyen du penétromètre. Fruits, I.F.A.C., France, 16(3):107-111.

FERREIRA, J,P. et alii., 1957. Enciclopédia dos Municípios Brasileiros. I.B.G.E. Rio de Janeiro, $28: 71-77 ; 193-195 ; 29: 446-450$.

HALLER, M.H., 1941. Fruit pressure testers and their practical aplications, U.S. Dep. Agri., Circular n. $627,1-22$.

; LUTZ, J.M. \& MALLISON, E.D., 1941. The relation of firmness to ripeness of eastern-grown apples. U.S. Dep. Agr. Circ. 579, 1-21.

- ; HARDING, P.L. \& ROSE, D.H., 1932. The interrelation of firmness, dry weight, and respiration in strawberries. Amer. Soc. Hort. Sci. Proc., 29:330-334.

IZURIETA, D.B., 1970. Obtenção de óleo de abacate. Tese apresentada para obtenção do título de Mestre em Engenharia de Alimentos. Faculdade de Tecnologia de Alimentos. Univ. Est. de Campinas, SP, 1-39.

LEOPOLD, A.C., 1964. Plant Growth and Development. McGraw-Hill Book Company. New York, 1-466.

MONTENEGRO, H.W.S., 1960. A cultura do abacateiro. Ed. Melhoramentos. Série Criação e Lavoura nọ î, São Paulo, 1-102.

SACHER, J.A., 1962. Relations between chances in membrane permeability and the climateric in banana and avocado. Nature. MacMillan and Co. Ltd., New York, 195(4841) 577-578.

SETZER, J., 1966. Atlas Climático e Ecológico do Estado de São Paulo. Comissão Interestadual da Bacia do Paraná-Paraquai. São Paulo, 161.

STAHL, A.L., 1933. Changes in composition of Florida avocados in relation to maturity. University of Florida. Agricultural Experiment Station Gainesville, Florida, U.S.A., Bull. 259, 161. 
ULRICH, R., 1952. La vie des fruits. Masson et Cia. Editeura. Paris, France, 1-370.

WILEY, R.C. \& WORTHINGTON, O.J., 1955. Fresh fruit objective test to predict the quality of canned prunes. Food Techni., 9:381-384. 